\title{
Diffusion of active ingredients in textiles
}

\section{A three step multiscale model}

\author{
Tineke Goessens · Benny Malengier · Pei Li · Rob \\ De Staelen
}

Received: date / Accepted: date

\begin{abstract}
Most practical textile models are based on a two scale approach: a one-dimensional fiber model and a fabric model, see [1]. No meso-level is used in between, i.e. the yarn scale is neglected in this setup. For dense textile substrates this seems appropriate as the yarns connect everywhere, but for loose fabrics or scrims this approach cannot be kept. Specifically when one is interested in tracking an active component released by the fibers, the yarn level plays an important role. This is because the saturation vapor pressure will influence the release rate from the fibers, and its value will vary over the yarn cross-section. Therefore, in this work we present a three step multiscale model: the active component is tracked in the fiber, the yarn, and finally at the fabric level. At the fiber level a onedimensional reduction to a non-linear diffusion equation is performed, and solved on a as needed basis. At the yarn level both a two-dimensional or a one-dimensional model can be applied, and finally the yarn result is upscaled to the fabric level.
\end{abstract}

Keywords Diffusion · Textile modelling $\cdot$ Upscaling $\cdot$ Multiscale modelling $\cdot$ Controlled release

Mathematics Subject Classification (2000) 35K57

*Authors gratefully acknowledge the support of the European Commission, FP7, project number 228639

Tineke Goessens

E-mail: tineke.goessens@ugent.be

Benny Malengier

E-mail: bm@cage.ugent.be

Pei Li

E-mail: pli@cage.ugent.be

Rob H. De Staelen

E-mail: rob.destaelen@ugent.be

Department of Mathematical Analysis, Research Group $\mathrm{NaM}^{2}$, Faculty of Engineering and Architecture, Ghent University, Belgium 


\section{Introduction}

The diffusion of substances in and through polymers is studied in a large variety of engineering applications. It is found in settings where controlled release plays an important role such as drug delivery and their encapsulation in the medical context, polymer melts and the inflation of elastic membranes for the construction of plastic materials, architecture and building, transport of particles through polymer coated materials such as textiles and many others. In the latter textile context polymer coating and encapsulation of substances for application on fibers creates functional materials such as dyes, fragrances, phase change materials, smart polymers and nanomaterials found in sports, defense, health care, envi- ronmental pollution control, space, and even everyday use products like rain coats or floor mats.

We focus on the diffusion of a substance to the outer boundary of textiles that are coated with a polymer solution of the active ingredient (AI), e.g. a perfume or a healing substance. This substance can easily be replaced by other volatiles. Based on the results of this study an inverse problem is encountered and once solved it can answer the question of how much of the AI has to be present on the textile fiber, so the concentration at the outer boundary of the textile stays high enough for as long as possible to be effective (e.g. have a noticeable odor for humans, a healing effect ...).

Models and algorithms for standard multilayer systems were considered [1,8,5,3]. These are now extended to the needs that have arisen during the research on the polymer finishes. The application in mind has the purpose to track the diffusion of an active component released by the fibers of a scrim, e.g. a gauze bandage. For textile substrates with an open structure like these scrims the previous approach is no longer suitable. This is because a scrim consists of loosely woven yarns so the release of the active component from the scrim actually equals the release of the component from the yarns. Furthermore the number of fibers and the configuration of these fibers in the yarn plays a role. Finally, the vapor pressure of the active component in the gaps of the yarn will influence the release rate from the fibers and varies over the yarn cross-section. As a conclusion we could say that a meso-level model that describes the release of the active component in the yarn cross-section is needed. Hence, with 'multiscale modelling' we indicate the full coupling between three levels of modelling, the micro-level which in practice could be the fibers, a meso-level corresponding to the yarns, and the macro-level, or thus the level of the total fabric. This approach gives the possibility to consider a complete multiscale model for volatiles and to optimize for fiber-yarn layouts and to obtain a better understanding of the life cycle of the textile. It was decided to not consider the effects of water and heat, as these don't play a major role in the use-scenario's of the finished fabrics at the moment.

The models have been developed and solved using the programming language Python in a toolbox called STICK (Sophisticated Textile Information Computation Kit). A full coupling between the three scales is present and the effect of different micro and meso-level layouts can be determined.

\section{Multiscale model}

\subsection{Setting}

In this study treated scrims are considered. To model this application we make a distinction on three levels of the scrim. First we model the fiber with a coating containing an active ingredient (AI). To this end the fiber will be seen as a cylindrical object. The boundary 
conditions depend on the chosen textile and the void space characteristics. Secondly we model the yarn, a porous structure built out of fibers, upscaling the outcome of the fiber model using an volume averaging technique. This yarn level can be developed in two ways, a two-dimensional model or a one-dimensional cylindrical model. The third model represents the scrim or fabric itself, with its environment, again using an upscaling method to calculate the overall properties of the fabric using the yarn properties.

\subsection{The micro-level}

If we consider the fiber as a long cylinder we can choose to work with a cylindrical coordinate system in which diffusion is everywhere radial. Diffusion of the AI in the fiber is then described by

$$
\frac{\partial C_{f}}{\partial t}=\frac{1}{r}\left\{\frac{\partial}{\partial r}\left(r D_{f} \frac{\partial C_{f}}{\partial r}\right)+\frac{\partial}{\partial \theta}\left(\frac{D_{f}}{r} \frac{\partial C_{f}}{\partial \theta}\right)+\frac{\partial}{\partial z}\left(r D_{f} \frac{\partial C_{f}}{\partial z}\right)\right\},
$$

where $C_{f}$ is the concentration of the $\mathrm{AI}$ in the fiber and $D_{f}$ is the diffusion coefficient of the $\mathrm{AI}$ in the fiber. According to a radial symmetric diffusion in a long cylinder [2] concentration is a function of the radial position $r$ and time $t$ only, so azimuth $\theta$ and height $z$ can be ignored and the diffusion equation becomes

$$
\frac{\partial C_{f}(r, t)}{\partial t}=\frac{1}{r} \frac{\partial}{\partial r}\left(r D_{f} \frac{\partial C_{f}(r, t)}{\partial r}\right), 0 \leq r \leq R_{f},
$$

with boundary condition at the fiber radius $R_{f}$

$$
\nabla C_{f}=\alpha\left(C^{*}(T)-C_{s}(t)\right) H\left(C-C_{b}, C^{*}(T)-C_{s}(t)\right) .
$$

Here, $C^{*}(T)$ is the equilibrium concentration at temperature $T, C_{s}$ is the concentration of the volatile around the surface of the fiber, and $H(x, y)$ is defined as the Heaviside function $H(x)$ if $y>0$, otherwise it is the identity, [6]. The diffusion coefficient $D_{f}$ can be concentration dependent, e.g. of the form $D_{f}\left(C_{f}\right)=D_{f_{0}} \exp \left(-c C_{f}\right)$. The reason for this $\mathrm{BC}$ is the fact that release from the fibers slows down if more of the component is in the air surrounding the fiber. This model has been solved using both a finite differences approach (see Fig. 1) and the method of lines (MOL) based on the finite volume method (FVM) (see Fig. 2).

For the MOL method the set-up is given in figure 3. We divide the domain into $n$ cells, the representative elementary volumes, $\Omega_{i}=\left[r_{i}, r_{i+1}\right]$, with $i=0, \ldots, n-1$ and use the following notation

$$
\begin{aligned}
& (\Delta r)_{i}^{+}=r_{i}-r_{i-1}, \quad r_{i}^{+}=r_{i+\frac{1}{2}}=r_{i}+\frac{(\Delta r)_{i}^{+}}{2} \\
& (\Delta r)_{i}^{-}=r_{i+1}-r_{i}, \quad r_{i}^{-}=r_{i-\frac{1}{2}}=r_{i}-\frac{(\Delta r)_{i}^{-}}{2}
\end{aligned}
$$

From now onwards we denote $C_{f}$ by $u$ and abbreviate $\frac{\partial}{\partial x}$ by $\partial_{x}$, mutatis mutandis. Set $w=u r$, then equation (1) becomes

$$
\partial_{t} w=\partial_{r}\left(r D \partial_{r} \frac{w}{r}\right),
$$




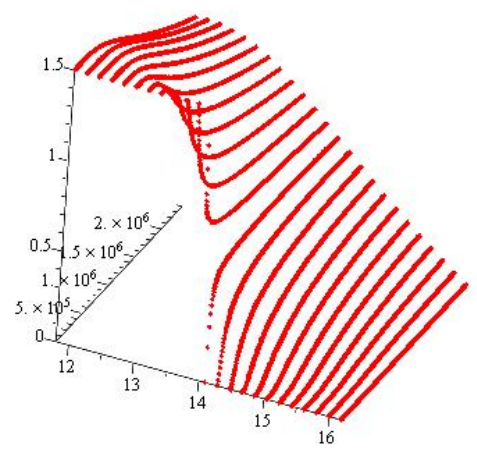

(a) Pointplot of solution for the AI

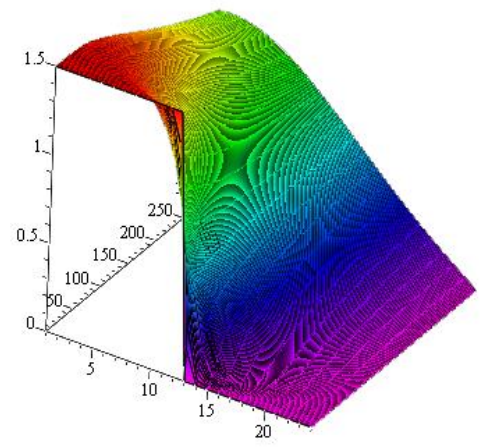

(b) Fitted surface through pointsolution for the AI

Fig. 1 The diffusion of the AI in time using finite difference method where the AI is directly at the fiber surface and taken to be $1500 \mathrm{mg} / \mathrm{m}^{3}$ at $t=0$

where $D=D_{0} e^{-c \frac{w}{r}}$. When we integrate over the domain $\Omega=[L, R]$ we get

$$
\int_{\Omega} \partial_{t} w \mathrm{~d} r=\left[r D \partial_{r} \frac{w}{r}\right]_{L}^{R}
$$

We denote the scaled concentration at time $t$ on space location $r_{i}$ as $w_{i}(t)$ and the scaled concentration in the centers of the adjacent cells by $w_{i}^{-}$and $w_{i}^{+}$in $\Omega_{i-1}$ and $\Omega_{i}$ respectively. Doing so we calculate the flux on the edge $r_{i}$ as

$$
\begin{aligned}
\int_{\Omega_{i}} \partial_{t} w_{i}(t) \mathrm{d} r=\partial_{t} w_{i}^{+} \int_{\Omega_{i}} \mathrm{~d} r & =\left[r D \partial_{r} \frac{w}{r}\right]_{r_{i}}^{r_{i+1}} \\
& =\left.r_{i+1} D_{i+1}\left(\partial_{r} \frac{w}{r}\right)\right|_{r_{i+1}}-\left.r_{i} D_{i}\left(\partial_{r} \frac{w}{r}\right)\right|_{r_{i}}
\end{aligned}
$$

These term are the fluxes along edge $r_{i+1}$ and $r_{i}$, respectively. For the flux along edge $r_{i}$ we have

$$
\begin{aligned}
\text { flux }_{\text {edge }_{i}} & =\left.r_{i} D_{i}\left(\partial_{r} \frac{w}{r}\right)\right|_{r_{i}} \\
& =\frac{r_{i}}{(\Delta r)_{i}^{+}+(\Delta r)_{i}^{-}}\left\{D_{0, i}^{+} \exp \left(-c \frac{w_{i}^{+}}{r_{i}^{+}}\right)+D_{0, i}^{-} \exp \left(-c \frac{w_{i}^{-}}{r_{i}^{-}}\right)\right\}\left(\frac{w_{i}^{+}}{r_{i}^{+}}-\frac{w_{i}^{-}}{r_{i}^{-}}\right)
\end{aligned}
$$

where we have taken the average diffusion coefficient of the adjacent cell centers. According to the above derivation we get

$$
\partial_{t} w_{i}^{+}=\left(\text {flux }_{\mathrm{edge}_{i+1}}-\text { flux }_{\mathrm{edge}_{i}}\right) \frac{1}{(\Delta r)_{i}^{+}},
$$

which can be easily implemented in Python. 


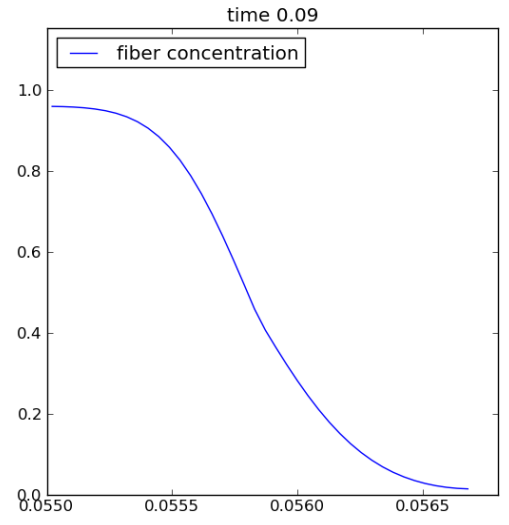

(a) $\mathrm{AI}$ concentration in the fiber after $0.09 \mathrm{~s}$

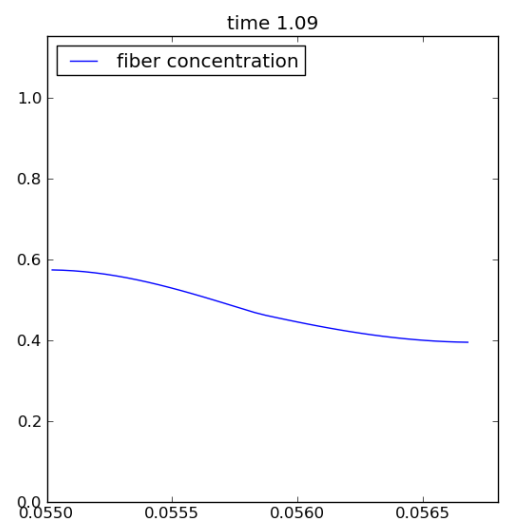

(c) AI concentration in the fiber after $1.09 \mathrm{~s}$

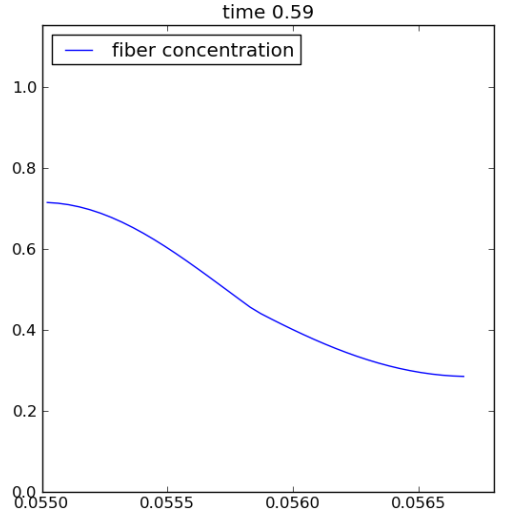

(b) AI concentration in the fiber after $0.59 \mathrm{~s}$

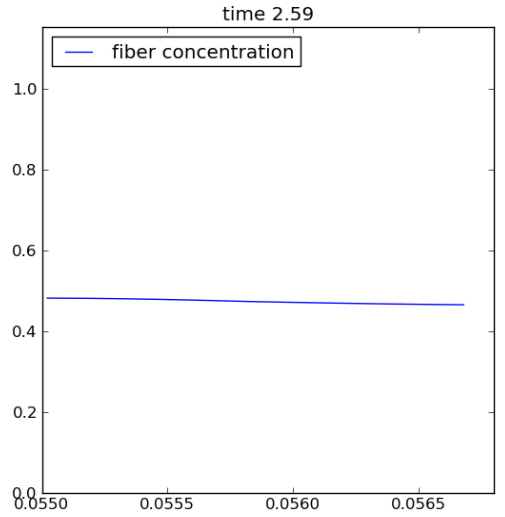

(d) AI concentration in the fiber after $2.59 \mathrm{~s}$

Fig. 2 The diffusion of the AI in time using MOL where the AI is directly at the fiber surface and taken to be $961 \mathrm{mg} / \mathrm{m}^{3}$ at $t=0$

\subsection{The meso-level}

Based on the model prescribed in [1], we choose to work with cylindrical coordinates $(r, \theta, z)$. By acknowledging there is no movement in the $\theta$ and $z$ direction we can work in only one dimension. The governing model for the concentration of the AI on the yarn level then is, according to the radial diffusion equation in a cylinder,

$$
\frac{\partial C_{y}(r, t)}{\partial t}=\frac{1}{r} \frac{\partial}{\partial r}\left(r \frac{D_{y}}{\tau_{y}} \frac{\partial C_{y}(r, t)}{\partial r}\right)+\Gamma(r, t) .
$$

The term $\Gamma$ in the equation above is a source term that describes the amount of AI coming out of a cross section of the fibers into the yarn air space. It is calculated by upscaling the concentration of AI on the boundary of a fiber using the volume averaging technique [9]. 


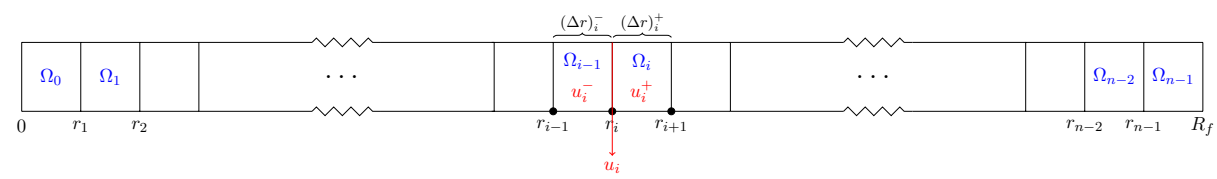

Fig. 3 The space discretization for MOL in a general fiber

The diffusion coefficient $D_{y}$ is that for diffusion of the AI through air. The tortuosity of the yarn $\tau_{y}$ is unknown, but can be estimated from a two-dimensional model which will be discussed further on.

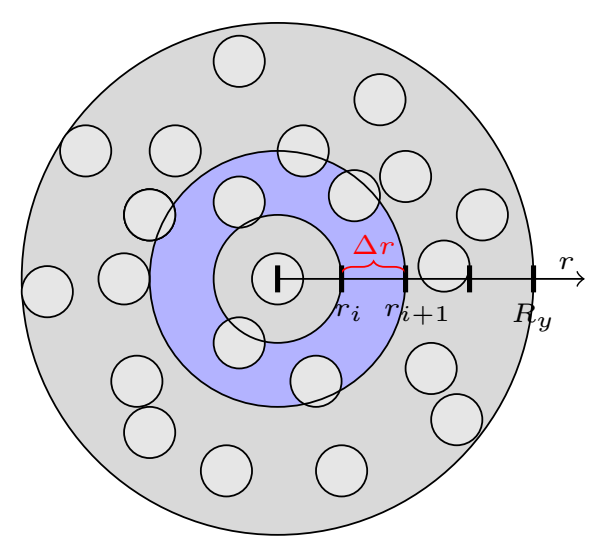

Fig. 4 Yarn discretization in cylindrical coordinates

In order to solve this model we divide the radial space in $M$ intervals $\left[r_{i}, r_{i+1}\right]$ with length $\Delta r=R_{y} / M$, see Fig. 4 , where $R_{y}$ is the yarn radius. If afterwards we also consider the $\theta$ coordinate we get concentric circles for each interval. In each of these shells we consider $n$ fibers. Using the fact that the area of such a shell between $\left[r_{i}, r_{i+1}\right]$ is $\pi\left(r_{i+1}^{2}-r_{i}^{2}\right)$ and the area of a fiber intersection with radius $R_{f}$ is $\pi R_{f}^{2}$ we get

$$
\varepsilon_{f}=\frac{n R_{f}^{2}}{\Delta\left(r^{2}\right)_{i}}
$$

for the volumetric fraction of fibers. The area of a yarn intersection with radius $R_{y}$ is $\pi R_{y}^{2}$. If we have $m$ fibers per yarn the fiber volumetric fraction also can be expressed as

$$
\varepsilon_{f}=\frac{m R_{f}^{2}}{R_{y}^{2}} .
$$

This means we can calculate the number of fibers in a shell $\left[r_{i}, r_{i+1}\right]$ as

$$
n=\frac{m \Delta\left(r^{2}\right)_{i}}{R_{y}^{2}} .
$$


In every timestep and space interval $\left[r_{i}, r_{i+1}\right]$ we solve one fibermodel using MOL based upon a FVM, assuming all $n$ fibers in the same shell satisfy the same boundary condition for the outer flux

$$
\frac{\partial C_{f}}{\partial r}=S_{y} h_{b \rightarrow f}\left(C^{*}(T)-C_{y}\left(r_{i+1 / 2}, t\right)\right) H\left(C-C_{b}, C^{*}(T)-C_{y}\left(r_{i+1 / 2}, t\right)\right),
$$

and the same initial condition $C_{y}\left(r_{i+1 / 2}, t\right)$, where $S_{y}$ is the surface area of the yarn cross section, $h_{b \rightarrow f}$ is the mass transfer coefficient for the AI from bounded to free, $C^{*}(T)$ and $H(x, y)$ are the equilibrium concentration and modified Heaviside function as explained earlier, [6]. We then take the volume average of the resulting surface concentration $C_{f}(R, r, t)$ to find the source term

$$
\Gamma\left(r_{i+1 / 2}, t\right)=\frac{n}{V} \int_{r_{i}}^{r_{i+1}} C_{f}(R) r d r .
$$

where $V$ is the shell area $\pi\left(r_{i+1}^{2}-r_{i}^{2}\right)$. Consequently we find

$$
\Gamma\left(r_{i+1 / 2}, t\right)=n \frac{C_{f}\left(R, r_{i}, t\right)+C_{f}\left(R, r_{i+1}, t\right)}{4 \pi} .
$$

Another way of solving this problem is using a 2D diffusion model of the $2 \mathrm{D}$ yarn cross-section containing fibers. The only the $z$ direction is neglected, and we write in $(x, y)$ coordinates

$$
\frac{\partial C_{y}(x, y, t)}{\partial t}=\nabla \cdot\left(D_{y} \nabla C_{y}(x, y, t)\right),
$$

where the tortuosity $\tau_{y}$ needed in the $1 \mathrm{D}$ model is no longer necessary. This model takes as a domain the circle circumscribing the yarn intersection with the fiber intersection circles cut out. On each inner boundary that arises like this a certain concentration of the AI is introduced as a flux boundary condition. This concentration can be calculated by dividing the time frame into steps $\Delta t$ and solving a fiber model on each step

$$
\frac{\partial C_{f}(x, y, t)}{\partial t}=\nabla \cdot\left(D_{f} \nabla C_{f}(x, y, t)\right),
$$

with boundary condition

$$
\nabla C_{f}=\alpha\left(C^{*}(T)-C_{y}(x, y, t)\right) H\left(C-C_{b}, C^{*}(T)-C_{y}(x, y, t)\right) .
$$

This 2D model is solved using FVM which has been implemented via the FiPy package [4] and can be used to estimate the tortuosity $\tau_{y}$ for the much faster 1D model described above.

\subsection{The macro-level}

For a scrim we can consider a model using the assumption that the yarns are infinitely long and the scrim is infinitely big for an observer close enough to t. The scrim can be visualized as a rectangular grid in 2D, with holes of width $d_{W}$ and height $d_{H}$. We thus consider an infinite net in the $Y Z$ plane, with an observer in $\left(x_{0}, 0,0\right)$, see Fig. 5 and the Cauchy problem for $-\infty<x, y, z<+\infty$,

$$
\begin{aligned}
\frac{\partial C}{\partial t}(x, y, z, t) & =D_{g} \Delta C+\Phi(x, y, z, t) \\
& =D_{g}\left(\frac{\partial^{2} C}{\partial x^{2}}+\frac{\partial^{2} C}{\partial y^{2}}+\frac{\partial^{2} C}{\partial z^{2}}\right)+\Phi(x, y, z, t)
\end{aligned}
$$


with initial condition $C=f(x, y, z)$ at $t=0$. Here the source term $\Phi(x, y, z, t)$ is the amount of AI coming out of the yarn at position $(x, y, z)$ at time $t$. Due to symmetry, we can consider the $X Y$ plane for the vertical yarns, and the $X Z$ plane for the horizontal yarn. Doing so one derivative becomes zero each time and the problem can be solved in $2 \mathrm{D}$ instead of $3 \mathrm{D}$, i.e. separately for vertical and horizontal yarns.

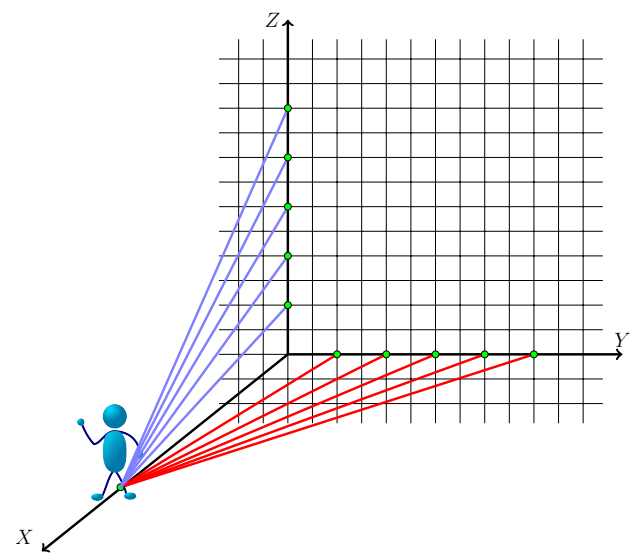

Fig. 5 The observer at distance $x_{0}$ from the net

For a vertical yarn at position $y=y_{v}$ the amount of AI at $t=0$ is taken to be the delta function scaled with the flux coming out of a yarn cross section, denoted by $F_{y_{v}}(0)$. Using the symmetry we know that $C_{z z}=0$. We then arrive at a $2 \mathrm{D}$ Cauchy problem of the form

$$
\frac{\partial C}{\partial t}(x, y, t)=D_{g}\left(\frac{\partial^{2} C}{\partial x^{2}}+\frac{\partial^{2} C}{\partial y^{2}}\right)+\Phi(x, y, t), \quad-\infty<x, y<+\infty,
$$

subject to

$$
C(x, y, 0)=F_{y_{v}}(0) \delta(x) \delta\left(y-y_{v}\right),
$$

where $D_{g}$ is the diffusion coefficient of the compound in air and we assume a source at $\left(0, y_{v}\right)$. The source term is

$$
\Phi(x, y, t)=F_{y_{v}}(t) \delta(x) \delta\left(y-y_{v}\right),
$$

where $F_{y_{v}}(t)$ is the amount of AI coming from a cross section of the yarn at $y=y_{v}$ at time $t$. The solution of this Cauchy problem for a vertical yarn is [7]

$$
C_{v}(x, y, t)=\frac{F_{y_{v}}(0)}{4 \pi D_{g} t} \exp \left[-\frac{x^{2}+\left(y-y_{v}\right)^{2}}{4 D_{g} t}\right]+\int_{0}^{t} \frac{F_{y_{v}}(\tau)}{4 \pi D_{g}(t-\tau)} \exp \left[-\frac{x^{2}+\left(y-y_{v}\right)^{2}}{4 D_{g}(t-\tau)}\right] \mathrm{d} \tau .
$$

By discretizing the past time period $[0, t]$ into $N$ timesteps and using the following estimation in each time interval for a given function $g(t)$

$$
\int_{(k-1) \Delta t}^{k \Delta t} g(\tau) F_{y_{v}}(\tau) \mathrm{d} \tau \approx F_{y_{v}}(k \Delta t) \int_{(k-1) \Delta t}^{k \Delta t} g(\tau) \mathrm{d} \tau=F_{y_{v}, k \Delta t} \int_{(k-1) \Delta t}^{k \Delta t} g(\tau) \mathrm{d} \tau,
$$


we can calculate the solution for a vertical yarn at $y=y_{v}$ as

$$
\begin{aligned}
C_{v}(x, y, t)= & \frac{F_{y_{v}}(0)}{4 \pi D_{g} t} \exp \left[-\frac{x^{2}+\left(y-y_{v}\right)^{2}}{4 D_{g} t}\right] \\
& +\sum_{k=1}^{N} \frac{F_{y_{v}, k \Delta t}}{4 \pi D_{g}}\left[E_{1}\left(-\frac{x^{2}+\left(y-y_{v}\right)^{2}}{4 D_{g}((k-1) \Delta t-t)}\right)-E_{1}\left(-\frac{x^{2}+\left(y-y_{v}\right)^{2}}{4 D_{g}(k \Delta t-t)}\right)\right] .
\end{aligned}
$$

where $E_{1}(\cdot)$ is the exponential integral function defined by

$$
E_{1}(z)=\int_{z}^{+\infty} \frac{e^{-} t}{t} \mathrm{~d} t
$$

For a horizontal yarn at position $z=z_{h}$ a similar problem and initial condition holds and an analogue solution $C_{h}(x, z, t)$ is met.

We suppose that the yarns are on such distance from one another that they do not influence each other. By doing so we can take the total solution of problem (2) to be a superposition of the separate solutions for all vertical and horizontal yarns. If we take the number of vertical and horizontal yarns to be $n$ and $m$ respectively we get, with $y_{v}^{n}$ the $n$ 'th vertical yarn, i.e. the yarn with Cartesian equation $y=n d_{W}$ and $z_{h}^{m}$ the $m$ 'th horizontal yarn, i.e. with Cartesian equation $z=m d_{H}$,

$$
\begin{aligned}
C(x, y, z, t) & =\sum_{n} C_{v}(x, y, t)+\sum_{m} C_{h}(x, z, t) \\
& =\sum_{n}\left\{\frac{F_{y_{v}^{n}}(0)}{4 \pi D_{g} t} \exp \left(-\frac{x^{2}+\left(y-y_{v}^{n}\right)^{2}}{4 D_{g} t}\right)\right. \\
& \left.+\sum_{k=1}^{N} \frac{F_{y_{v}^{n}, k \Delta t}}{4 \pi D_{g}}\left[E_{1}\left(-\frac{x^{2}+\left(y-y_{v}^{n}\right)^{2}}{4 D_{g}((k-1) \Delta t-t)}\right)-E_{1}\left(-\frac{x^{2}+\left(y-y_{v}^{n}\right)^{2}}{4 D_{g}(k \Delta t-t)}\right)\right]\right\} \\
& +\sum_{m}\left\{\frac{F_{z_{h}^{m}}(0)}{4 \pi D_{g} t} \exp \left(-\frac{x^{2}+\left(z-z_{h}^{m}\right)^{2}}{4 D_{g} t}\right)\right. \\
& \left.+\sum_{k=1}^{N} \frac{F_{z_{h}^{m}, k \Delta t}}{4 \pi D_{g}}\left[E_{1}\left(-\frac{x^{2}+\left(z-z_{h}^{m}\right)^{2}}{4 D_{g}((k-1) \Delta t-t)}\right)-E_{1}\left(-\frac{x^{2}+\left(z-z_{h}^{m}\right)^{2}}{4 D_{g}(k \Delta t-t)}\right)\right]\right\} .
\end{aligned}
$$

For an observer at position $\left(x_{0}, 0,0\right)$ the amount of observed AI coming out of the scrim will be,

$$
\begin{aligned}
C\left(x_{0}, 0,0, t\right) & =\frac{1}{4 \pi D_{g}} \sum_{n=-\infty}^{+\infty}\left\{\frac{F_{n d_{W}}(0)}{t} \exp \left(-\frac{r_{v, n}^{2}}{4 D_{g} t}\right)\right. \\
& \left.+\sum_{k=1}^{N} F_{n d_{W}, k \Delta t}\left[E_{1}\left(-\frac{r_{v, n}^{2}}{4 D_{g}((k-1) \Delta t-t)}\right)-E_{1}\left(-\frac{r_{v, n}^{2}}{4 D_{g}(k \Delta t-t)}\right)\right]\right\} \\
& +\frac{1}{4 \pi D_{g}} \sum_{m=-\infty}^{+\infty}\left\{\frac{F_{m d_{H}}(0)}{t} \exp \left(-\frac{r_{h, m}^{2}}{4 D_{g} t}\right)\right. \\
& \left.+\sum_{k=1}^{N} F_{m d_{H}, k \Delta t}\left[E_{1}\left(-\frac{r_{h, m}^{2}}{4 D_{g}((k-1) \Delta t-t)}\right)-E_{1}\left(-\frac{r_{h, m}^{2}}{4 D_{g}(k \Delta t-t)}\right)\right]\right\},
\end{aligned}
$$


which leads to

$$
\begin{aligned}
C\left(x_{0}, 0,0, t\right) & =\frac{1}{4 \pi D_{g}} \sum_{n=-\infty}^{+\infty}\left\{\frac{F_{n d_{W}}(0)}{t} \exp \left(-\frac{r_{v, n}^{2}}{4 D_{g} t}\right)-\sum_{k=1}^{N} F_{n d_{W}, k \Delta t} \int_{\frac{r_{v, n}^{2}}{4 D_{g}(k \Delta t-t)}}^{\frac{r_{v, n}^{2}}{4 D_{g}}} \frac{e^{-u}}{u} d u\right\} \\
& +\frac{1}{4 \pi D_{g}} \sum_{m=-\infty}^{+\infty}\left\{\frac{F_{m d_{H}}(0)}{t} \exp \left(-\frac{r_{h, m}^{2}}{4 D_{g} t}\right)-\sum_{k=1}^{N} F_{m d_{H}, k \Delta t} \int_{\frac{r_{h, m}^{2}}{4 D_{g}(k \Delta t-1)}}^{\frac{r_{h, t}^{2}}{4(k-1) \Delta t-t)}} \frac{e^{-u}}{u} d u\right\}
\end{aligned}
$$

where $r_{v, n}^{2}=x_{0}^{2}+n^{2} d_{W}^{2}$ and $r_{h, m}^{2}=x_{0}^{2}+m^{2} d_{H}^{2}$ are the shortest distances to the yarn in question, and where we used $y_{v}^{n}=n d_{W}$ and $z_{h}^{m}=m d_{H}$.

\section{Results and conclusion, future work}

A three-scale model consisting of a micro-, meso- and macrolevel was constructed, implemented in Python and solved. A full coupling was established between the different levels in order to be able to control effects from layout and environmental changes. The outcome of the implemented three-step model is in accordance with the expected outcome. Further validation should be done with obtained experimental data. With the information at hand an inverse problem can now be solved to establish some estimates for the diffusion coefficients in the model and some conclusions can be made about which initial concentrations should be applied on the fibers to achieve a sufficient quality of the application under consideration, e.g. a noticeable odor at a particular distance from the scrim. The tortuosity $\tau_{y}$ in the rather fast 1D meso-model can be estimated from the outcome of the 2D model. The fabric level can be further extended to a model for dense textile substrates. A comparison then can be made to see if also for this kind of fabrics the yarn level is needed.

Acknowledgements Authors gratefully acknowledge the support of the European Commission, FP7, project number 228639

\section{References}

1. J. Fan C. Ye, H. Huang and W. Sun. Numerical study of heat and moisture transfer in textile materials by a finite volume method. Communications in Computational Physics, 4(4):929-948, 2008.

2. J. Crank. The Mathematics of Diffusion. Clarendon Press Oxford, 1979.

3. J. Fan and X. Wen. Modeling heat and moisture transfer through fibrous insulation with phase change and mobile condensates. International Journal of Heat and Mass Transfer, 45(19):4045-4055, 2002.

4. J. E. Guyer, D. Wheeler, and J. A. Warren. FiPy: Partial differential equations with Python. Computing in Science \& Engineering, 11(3):6-15, 2009.

5. Z. Luo J. Fan and Y. Li. Heat and moisture transfer with sorption and condensation in porous clothing assemblies and numerical simulation. International Journal of Heat and Mass Transfer, 43(16):29893000, 2000.

6. Y. Li and Q. Zhu. Simultaneous heat and moisture transfer with moisture sorption, condensation, and capillary liquid diffusion in porous textiles. Textile Research Journal, 73(6):515-524, 2003.

7. A.D. Polyanin. Handbook of Linear Partial Differential Equations for Engineers and Scientists. Chapman \& Hall/CRC, 2002

8. Z. Wang. Heat and moisture transfer and clothing thermal comfort. PhD thesis, Hong Kong Polytechnic University, 2002.

9. S. Whitaker. The Method of Volume Averaging. Theory and Applications of Transport in Porous Media. Kluwer Academic, 1999. 